cardiac arrests requiring chest compressions, defibrillation, or both. As expected, fewer children than adults experienced VF as their first documented cardiac arrest, although asystole and pulseless activity were the most common first events in both groups. Overall survival to hospital discharge following pulseless cardiac arrest was, surprisingly, higher among children than adults (27\% vs $18 \%)$. After adjustment, only first documented pulseless cardiac arrest rhythm showed significant association with differential survival to hospital discharge.

The improved survival in children was possibly related to early, aggressive resuscitationchildren were treated in intensive care more frequently than adults to monitor for respiratory failure and circulatory shock. The authors urge that training be given to aid early identification of these causes in children and to differentiate shockable from nonshockable rhythms.

Pippa Murdie

Original article Nadkarni VM et al. (2006) First

documented rhythm and clinical outcome from in-hospital cardiac arrest among children and adults. JAMA 295: 50-57

\section{STEMI: is 30 -day mortality rate associated with gross national income?}

A study recently reported in the European Heart Journal investigated whether 30-day mortality in patients with ST-segment elevation myocardial infarction (STEMI) is associated with the gross national income (GNI) of their country.

Patients with STEMI from five large multicenter trials $(n=50,310)$ were included in the analysis. Patients were recruited over 5 years from 53 countries in 5 continents, and had been enrolled within 6-12h of symptom onset. Countries were divided into three GNI groups: low (<US\$2,900 per capita), medium (\$2,900-9,000 per capita) and high $(>\$ 9,000$ per capita).

Significant differences in 30-day mortality were observed between groups: $12.1 \%$ for low GNI countries, 9.4\% for medium GNI countries and $4.9 \%$ for high GNI countries $(P<0.001$ for all comparisons). Continuous measurements of GNI showed an inverse relationship with 30 -day mortality up to approximately $\$ 20,000$ per capita; above this value, the mortality rate remained relatively constant. When adjustments were made for mortality risk factors, this inverse relationship was diminished, but mortality risk remained higher than expected in the low and medium GNI countries, and lower than expected in the high GNI countries.

Orlandini et al. acknowledge the study's limitations but conclude that 30-day mortality in STEMI patients in clinical trials increases with decreasing GNI. This pattern is partially influenced by differing baseline characteristics, but other factors also contribute. The authors urge that future studies include a wider range of countries with different GNIs, so that trial results are more generally applicable.

Rebecca Ireland

Original article Orlandini A et al. (2006) Outcomes of patients in clinical trials with ST-segment elevation myocardial infarction among countries with different gross national incomes. Eur Heart J [doi: 10.1093/eurheartj/ehi701]

\section{Increased fruit and vegetable intake cuts risk of stroke}

Epidemiological studies have reported a link between fruit and vegetable consumption and a reduced incidence of stroke, cardiovascular disease and some cancers. Individual studies of stroke, however, have varied in their measurements of exposure and disease, making findings hard to compare. He et al. have, therefore, undertaken a meta-analysis of prospective cohort studies to quantify the association between this disorder and fruit and vegetable intake.

Altogether, 257,551 individuals were included in the meta-analysis, from nine independent cohorts: five from the US, three from Europe, and one from Japan. With an average serving calculated as $77 \mathrm{~g}$ for vegetables and $80 \mathrm{~g}$ for fruit, consumption was standardized into three categories of daily servings: fewer than three, three to five and more than five servings. Relative risks or hazard ratios were adjusted for confounding factors such as age, smoking and alcohol consumption.

Over a median follow-up of 13 years, a total of 4,917 stroke events occurred. Compared with people who consumed fewer than three portions of fruit and vegetables daily, the risk of stroke was $11 \%$ lower among those who ate three to five servings, and as much as $26 \%$ 